\title{
Occurrence of primary brain tumors in cochlear implant patients in Sweden between 1989 and 2014
}

This article was published in the following Dove Press journal: Clinical Epidemiology

\author{
Henrik Smeds ${ }^{1,2}$ \\ Jeremy Wales ${ }^{1,2}$ \\ Tiit Mathiesen ${ }^{3-6}$ \\ Mats Talbäck ${ }^{7}$ \\ Maria Feychting ${ }^{7}$ \\ 'Department of Otolaryngology, \\ Karolinska University Hospital, \\ Stockholm, Sweden; ${ }^{2}$ Department of \\ Clinical Science, Intervention and \\ Technology, Karolinska Institutet, \\ Stockholm, Sweden; ${ }^{3}$ Department \\ of Neurosurgery, Karolinska \\ University Hospital, Stockholm, \\ Sweden; ${ }^{4}$ Department of Clinical \\ Medicine, University of Copenhagen, \\ Copenhagen, Denmark; ${ }^{5}$ Department \\ of Clinical Neuroscience, Karolinska \\ Institutet, Stockholm, Sweden; \\ ${ }^{6}$ Department of Neurosurgery, \\ Rigshospitalet, Copenhagen, Denmark; \\ ${ }^{7}$ Unit of Epidemiology, Institute of \\ Environmental Medicine, Karolinska \\ Institutet, Stockholm, Sweden
}

Purpose: Cochlear implants are widely used for hearing rehabilitation of deaf children with congenital deafness or adults with acquired severe-to-profound hearing loss. The sound processor antenna creates a radio frequency-electromagnetic field transmitting the sound signal to the implant, similar to that in a mobile phone. A recent case report suggested a relationship between cochlear implants and malignant glioma, and some epidemiological studies have suggested an increased glioma and acoustic neuroma risk associated with long hours of mobile phone use. An epidemiological study is warranted to evaluate such a relationship in patients with cochlear implants.

Patients and methods: To examine whether this chronic radio frequency-electromagnetic field signaling is associated with an increased brain tumor risk, a population-based cohort study was performed examining all 2,748 patients receiving a cochlear implant in Sweden during the years 1989-2014. In all, 3,169 surgeries were performed in the total cohort. The expected occurrence of glioma, meningioma, and acoustic neuroma in the patient cohort was calculated using specific national incidence rates in the Swedish population.

Results: Four patients were diagnosed with a brain tumor during follow-up, three of them having meningioma compared with 0.95 expected (standardized incidence ratio $=3.16,95 \%$ CI $0.65-$ 9.24), and one had glioma compared with 1.34 expected (standardized incidence ratio $=0.75$, 95\% CI 0.02-4.15). No case of acoustic neuroma was observed compared with 0.09 expected. Conclusion: In this study, we did not find support for concerns raised in a previous case report regarding a potentially higher risk of glioma. The number of brain tumors observed was well within the numbers expected from national incidence figures. Although this was a relatively small cohort with a limited follow-up time, it is the largest epidemiological study to date to address this concern.

Keywords: cochlear implants, glioblastoma, neural tumor, non-ionizing radiation, radio frequency-electromagnetic radiation

\section{Introduction}

A cochlear implant is an electric device that turns sound into electrical impulses. It has revolutionized the treatment of severe-to-profound deafness and remains the most successful neural prosthesis in the world, with more than 500,000 recipients implanted. It consists of an external part, the sound processor, and an internal implant, the receiver-stimulator, and the electrode array. Sound is sent through the skin as a frequency modulation signal to the implant by an antenna behind the ear. The coil of the implant picks up the signal and converts it into electrical impulses, and these are distributed to the cochlea corresponding to sound frequencies. Cochlear implants do
Correspondence: Henrik Smeds Department of Otolaryngology, Karolinska University Hospital, B53 ENT,

I4I86 Stockholm, Sweden

Tel +46858580000

Fax +468746755I

Email henrik.smeds@sll.se 
not restore hearing to normal but allow people that are deaf or with very profound hearing loss to perceive speech and other sounds with good results.

Cochlear implants have a long history of safety, with the most significant neurological side effect being bacterial meningitis, although this is rare. However, it has been suggested that radio frequency electromagnetic fields (RF-EMFs) generated by mobile phones and wireless devices may be carcinogenic, based on epidemiological evidence on glioma and acoustic neuroma. ${ }^{1}$ However, the evidence is not conclusive. ${ }^{2}$ Some epidemiological studies suggest that effects are confined to persons with many hours of mobile phone use, ${ }^{3,4}$ whereas others have not been able to confirm this. ${ }^{5}$

We have recently reported on two cases of glioblastoma in geographically unrelated patients, one from Sweden and the other from the USA, who had long-standing cochlear implants. ${ }^{6}$ Extrapolating from the discussions regarding mobile phone use, the two cases raised concerns that longterm exposure to low levels of RF-EMF emanating from the transcutaneous link of the cochlear implant could increase the risk of neurological tumors in patients with cochlear implants.

The risk of life-threatening side effects of a medical procedure, no matter how rare, should always be discussed with a patient before they undergo that procedure. This populationbased cohort study was designed to assess if patients that had undergone cochlear implantation had an increased risk of neural tumors at a population level. This would allow practitioners to comprehensively discuss and assess the possibility of an increased risk of tumors postoperatively for patients about to undergo implantation.

\section{Patients and methods}

The study is based on the Swedish population and health data registers, and the unique personal identification number assigned to all Swedish residents. Records of cochlear implant surgeries were extracted from the National Patient Register for in-patient care between 1989 and 2014. The Classification of Surgical Procedures used by the Swedish National Board of Health and Welfare was the basis for identification, with the code 2058 during the years 1989-1996 and DFE00 during 1997-2014. Cochlear implant patients were followed in the Swedish Cancer Register 1990-2015 from 1 year after their first implant surgery, to account for a minimal induction and latency period. They were censored at the date of the first diagnosis of glioma, meningioma, or acoustic neuroma; date of death; date of first emigration after their first cochlear implant surgery; or at the end of follow-up on 31 December 2015. Eligible cases were first occurrence of glioma, meningioma, or acoustic neuroma during the followup period. Tumors were classified according to definitions used in the Swedish Cancer Register: ICD-7 and WHO/ HS/CANC/24.1 prior to 1993; meningioma (ICD-7: 193.0 with histology codes 461, 466), glioma (193.0, 475-476, 485-486), and acoustic neuroma (193.0, 451, 456), and after 1992, the tumors were classified according to ICD$\mathrm{O} / 2$; meningioma (ICD-O/2: C70.0 with histology codes 9530-9539), glioma (C71, 9380-9481, 9505), and acoustic neuroma (C72.4, 9560.0, 9560.3). The study was approved by the regional ethical review board in Stockholm, Sweden. Non-identifiable data from national health data registers were made available to the research team by the National Board of Health and Welfare, after assessment of patient confidentiality and removal of all personal identifiers. No individual medical records were accessed during this study.

\section{Statistical analysis}

The expected number of glioma, meningioma, and acoustic neuroma cases in the cochlear implant cohort and standardized incidence ratios (SIR) for these tumors were calculated using sex, age (10-year categories), and calendar year (in 5 -year categories)-specific national incidence rates in the Swedish population as comparison. The expected number of tumors, point estimates, and CIs of SIR were calculated using command for indirect standardization in STATA 14 (StataCorp LP, College Station, TX, USA).

\section{Results}

According to the register, 3,169 cochlear implant operations were performed on 2,748 patients during this period; $14.3 \%$ (395) of patients had surgery during more than one admission. This included sequential bilateral implantation or revision surgeries, where 23 patients had three or more surgeries during this period. The patients were born between 1912 and 2014 and had had surgery at ages between less than 1 year and 89 years, $55 \%$ were females. Twenty-one percent of the patients had their first cochlear implant surgery before 5 years of age (Table 1 ).

We required tumors to be diagnosed $>1$ year after implant surgery to be considered as radiation induced. Seventeen patients were excluded from the study because they had been diagnosed with a brain tumor prior to their first cochlear implant surgery (between 3 months and 42 years earlier). All cochlear implant recipients undergo temporal bone imaging as a part of their preoperative investigations so these were not necessarily symptomatic. Eight of these patients were diagnosed with meningioma, two with glioma, and eight 
Table I Number of patients receiving a cochlear implant in 1989-20I4 and eligible for follow-up by year and age at surgery ( $\mathrm{n}=2,7 \mid 4)$

\begin{tabular}{|c|c|c|c|c|c|c|c|}
\hline & \multicolumn{2}{|c|}{ Number of patients } & \multicolumn{2}{|c|}{ Person-years } & \multicolumn{3}{|l|}{ Tumors } \\
\hline & Males & Females & Males & Females & Meningioma & Glioma & AN \\
\hline \multicolumn{8}{|l|}{ Time period } \\
\hline 1989-1994 & 43 & 51 & 54 & 60 & & & \\
\hline $1995-1999$ & 104 & 96 & 317 & 381 & & & \\
\hline 2000-2004 & 138 & 154 & 850 & 879 & & & \\
\hline 2005-2009 & 378 & 481 & $\mathrm{I}, 817$ & 2,077 & 2 & & \\
\hline 2010-2015 & 566 & 703 & 4,789 & 5,905 & 1 & 1 & \\
\hline Age (years) & \multicolumn{2}{|c|}{ At surgery } & \multicolumn{2}{|c|}{ During follow-up } & & & \\
\hline $0-4$ & 287 & 276 & 443 & 426 & & & \\
\hline $5-14$ & 91 & 95 & 1,638 & $|, 62|$ & & & \\
\hline $15-29$ & 37 & 77 & 785 & 867 & & & \\
\hline $30-59$ & 299 & 443 & 1,613 & 2,375 & 2 & 1 & \\
\hline $60+$ & 515 & 594 & 3,347 & 4,014 & $\mathrm{I}$ & & \\
\hline
\end{tabular}

Notes: Person-years at risk during follow-up 1990-2015 by calendar year and attained age. Number of brain tumors diagnosed during the follow-up period. Abbreviation: AN, acoustic neuroma.

with acoustic neuroma (one patient was diagnosed with both glioma and meningioma). One patient was diagnosed with acoustic neuroma on the same day as the cochlear implant operation and one patient was diagnosed with glioma 9 months after surgery. These two patients were also excluded. An additional 15 patients were censored within 1 year of operation and were also subsequently excluded from analysis. Twelve of these patients had died and three had emigrated within 1 year of surgery. The remaining 2,714 cochlear implant patients were eligible to be included in the study (Table 1). Side of the tumor as compared to the cochlear implant was not considered.

The cochlear implant cohort generated in total 17,129 person-years during the follow-up period. The mean followup time of all patients was 6.3 years (median 5.1 years, 25 th percentile $2.3,75$ th percentile 8.7 ). Of the eligible patients, four were diagnosed with a brain tumor during this time, between 3 and 17 years after their first cochlear implant surgery $(2.7,3.0,4.0$, and 17.3 years). Three of these tumors were meningioma compared with 0.95 expected $(\mathrm{SIR}=3.16$, 95\% CI 0.65-9.24), and one was a glioma compared with 1.34 expected (SIR $=0.75,95 \%$ CI $0.02-4.15$ ). No case of acoustic neuroma was observed compared with 0.09 expected.

\section{Discussion}

Contrary to the concerns raised by our recently published case report, ${ }^{6}$ we did not find an increased risk of malignant glioma, meningioma, or schwannoma in our cohort of patients with cochlear implants. The RF-EMFs generated by a cochlear implant are thus unlikely to induce tumors. Large epidemiological studies have failed to show a consistent increased risk of brain tumors in persons exposed to
RF-EMF. ${ }^{3,4}$ Some results indicated a potential effect associated with a large number of cumulative hours of phone use, although biases and errors could not be excluded as alternative explanations. ${ }^{3,4} \mathrm{~A}$ cochlear implant generates weaker RFEMF compared with mobile phones $(20-40 \mathrm{~mW}$ vs $2 \mathrm{~W})$ and operates at different frequencies (the majority of implants in Sweden use 5 or $12 \mathrm{MHz}$ compared with $900-5,800 \mathrm{MHz}$ for mobile phones). ${ }^{7}$ However, the maximum effect of $2 \mathrm{~W}$ from a mobile phone is for the second-generation phones (Global System for Mobile communications [GSM] 900 $\mathrm{MHz}$ ) when operating at maximum power. Adaptive power control makes the phone downregulate the output power to the lowest level possible in order to maintain communication, and reduces the emitted power by a factor of up to 1,000 for GSM phones and about 100,000,000 for Universal Mobile Telecommunications System (UMTS) phones. This gives an average reduction of $50 \%$ for GSM phones, and about two orders of magnitude further reduction for UMTS phones. ${ }^{2}$ In addition, GSM phones use Time Division Multiple Access, which means that the phone transmits only at regular intervals, ie $12 \%$ of the time. Furthermore, discontinuous transmission during voice calls gives a further reduction of the emitted power by an average of $30 \% .^{2}$ This means that the average emitted power during mobile phone use is $88 \mathrm{~mW}$ from a GSM $900 \mathrm{MHz}$ phone and $44 \mathrm{~mW}$ from a GSM 1800 $\mathrm{MHz}$ phone, whereas from a UMTS phone average emitted power is considerably lower. Thus, the exposure levels generated by a cochlear implant may be of the same order of magnitude as from a mobile phone. In addition, it could be argued that over time exposure from a cochlear implant will become significant as most cochlear implant users will use their implant constantly around 16 hours per day. 
The observed incidence of brain tumors in the cochlear implant cohort was well within the numbers expected, considering random variation, as reflected by the $95 \%$ CI. The number of cochlear implant patients is small and the follow-up relatively short. We cannot rule out that a more highly powered study would find weak associations between exposure and tumor incidence. Still, the only patient with a glioblastoma among the cochlear implant patients in Sweden was our one Swedish index case in the case report (the other was from the USA $)^{6}$ and the absence of additional patients is indicative of low risk. Various risk factors have been associated with glioma, ${ }^{8}$ but cochlear implants were only suggested in our previous report of two cases of glioblastoma after long-term cochlear implantation. ${ }^{6}$ In these cases, the anatomical relation to the implant antennas was compelling, but two patients are a very small proportion of the more than 500,000 cochlear implant recipients around the world. Furthermore, our present findings were based on the comprehensive population-based Swedish registers, ensuring complete follow-up, which is a strength. A more relevant objection could be that longer follow-up is necessary to account for tumor latency. Tumor latencies after ionizing radiation exposure are $>5-10$ years. ${ }^{9-11}$ For nonionizing radiation, there is no known biological mechanism for a potential carcinogenic effect, and the tumor latency is unknown. ${ }^{2-4}$ Studies on mobile phone use have reported on various latencies with no consistent pattern of results. $^{2-4}$ The latency of tumors induced by ionizing radiation is at least greater than 4 years, ${ }^{10,11}$ whereas the latency of tumors potentially induced from nonionizing radiation is unknown. Therefore, we excluded tumors detected before or during application of cochlear implants and tumors detected less than 1 year after surgery, as it was judged that tumors with such short latency could not have been caused by the exposure from the cochlear implant. This is consistent with the 1-year latency applied in studies of mobile phone use. ${ }^{2-4}$ This conservative cutoff for latency is defendable allowing false positive rather than false-negative observations of radiation induced tumors, where we considered this to be of primary importance in the present setting. In addition, the Interphone study found the strongest risk estimate for glioma among persons with many hours of mobile phone use accrued with short latency $\left(<5\right.$ years). ${ }^{3}$ Thus, the exposure from a cochlear implant in our study may be of a comparable intensity and duration. Our findings do not confirm the results in the Interphone study for glioma. Studies of meningioma in relation to mobile phone use have not indicated increased risks. ${ }^{1}$

A cochlear implant consists of two parts, an external sound processor with microphones and antenna and an internal receiver-stimulator that is beneath the skin and imbedded in the skull. Communication between these two parts is dependent on RF signaling that in turn generates an RF-EMF. There is significant debate in the literature around whether RF-EMF can result in an increased incidence of neurological tumors. A few case-control studies with self-reported histories of mobile phone use have reported considerable risk increases among mobile phone users primarily for glioma and acoustic neuroma, and also after short exposure latencies (reviewed by Scientific Committee on Emerging and Newly Identified Health Risks [SCENIHR]). ${ }^{2}$ However, cohort studies with independently collected exposure information have not found any increased risks, ${ }^{12-14}$ and incidence trend studies have not found changes in the brain tumor incidence that would correspond to an effect of RF exposure from mobile phone use. ${ }^{15,16}$ On the contrary, brain tumor incidence trends have been stable since the introduction of handheld mobile phones. Taken together, our negative findings in this study agree with a large body of epidemiological literature on RF-EMF exposure. ${ }^{2}$

\section{Conclusion}

Our study shows no relationship between cochlear implant use and the incidence of glioma or any other neural tumor. These epidemiological findings do not support an increased risk of neurological tumors in patients with cochlear implants, and, therefore a risk of malignancy need not be part of patient information or informed consent before cochlear implantation.

\section{Acknowledgments}

The study has not been presented elsewhere. The manuscript was approved by all the authors, of which J Wales is a native English speaker.

\section{Disclosure}

MF is vice chairman of the International Commission on Non-Ionizing Radiation Protection, an independent body setting guidelines for nonionizing radiation protection. She serves as advisor to a number of national and international public advisory and research steering groups concerning the potential health effects of exposure to non-ionizing radiation. The authors report no other conflicts of interest in this work.

\section{References}

1. IARC. Non-ionizing radiation. Part 2, Radiofrequency electromagnetic fields. IARC monographs on the evaluation of carcinogenic risks to humans. Vol. 102. International Agency for Research on Cancer: Lyon; 2013.

2. SCENIHR. Scientific Committee on Emerging and Newly Identified Health Risks: Potential health effects of exposure to electromagnetic fields $(E M F)$. Available from: http://ec.europa.eu/health/scientific_committees/ emerging/docs/scenihr_o_041.pdf;2015. Accessed August 15, 2017. 
3. Interphone Study Group. Brain tumour risk in relation to mobile telephone use: results of the INTERPHONE international case-control study. Int J Epidemiol. 2010;39(3):675-694.

4. Interphone Study Group. Acoustic neuroma risk in relation to mobile telephone use: results of the INTERPHONE international case-control study. Cancer Epidemiol. 2011;35(5):453-464.

5. Pettersson D, Mathiesen T, Prochazka M, et al. Long-term mobile phone use and acoustic neuroma risk. Epidemiology. 2014;25(2): 233-241.

6. Kalakoti P, Murray RD, Pettersson-Segerlind J, Smeds H, Nanda A. Cochlear implants in the etiopathogenesis of glioblastoma-an interesting observation or independent finding? Acta Neurochir. 2016;158(5): 907-912.

7. Zeng FG, Rebscher S, Harrison W, Sun X, Feng H. Cochlear implants: system design, integration, and evaluation. IEEE Rev Biomed Eng. 2008;1:115-142.

8. Ostrom QT, Bauchet L, Davis FG, et al. The epidemiology of glioma in adults: a "state of the science" review. Neuro Oncol. 2014;16(7): 896-913.

9. Fraser DK. Latency period of radiation-induced cancer. CMAJ. 2011;183(17):20172017; author reply 2017.
10. Nishio S, Morioka T, Inamura T, et al. Radiation-induced brain tumours: potential late complications of radiation therapy for brain tumours. Acta Neurochir. 1998;140(8):763-770.

11. Hall E. Radiobiology for the Radiologist. 5th ed. Philadelphia: Lippincott Williams \& Wilkins; 2000.

12. Frei P, Poulsen AH, Johansen C, Olsen JH, Steding-Jessen M, Schüz J. Use of mobile phones and risk of brain tumours: update of Danish cohort study. BMJ. 2011;343:d6387.

13. Benson VS, Pirie K, Schüz J, et al. Mobile phone use and risk of brain neoplasms and other cancers: prospective study. Int J Epidemiol. 2013;42(3):792-802.

14. Benson VS, Pirie K, Schüz J, Reeves GK, Beral V, Green J. Authors' response to: the case of acoustic neuroma: comment on mobile phone use and risk of brain neoplasms and other cancers. Int J Epidemiol. 2014;43(1):275.

15. Deltour I, Auvinen A, Feychting M, et al. Mobile phone use and incidence of glioma in the Nordic countries 1979-2008: consistency check. Epidemiology. 2012;23(2):301-307.

16. Little MP, Rajaraman P, Curtis RE, et al. Mobile phone use and glioma risk: comparison of epidemiological study results with incidence trends in the United States. BMJ. 2012;344:e1147.
Clinical Epidemiology

\section{Publish your work in this journal}

Clinical Epidemiology is an international, peer-reviewed, open access, online journal focusing on disease and drug epidemiology, identification of risk factors and screening procedures to develop optimal preventative initiatives and programs. Specific topics include: diagnosis, prognosis, treatment, screening, prevention, risk factor modification,

Submit your manuscript here: https://www.dovepress.com/clinical-epidemiology-journal

\section{Dovepress}

systematic reviews, risk and safety of medical interventions, epidemiology and biostatistical methods, and evaluation of guidelines, translational medicine, health policies and economic evaluations. The manuscript management system is completely online and includes a very quick and fair peer-review system, which is all easy to use. 